\title{
Power-Domain Based Dynamic Millimeter-Wave Spectrum Access Techniques for In-Building Small Cells in Multioperator Cognitive Radio Networks toward 6G
}

\author{
Rony Kumer Saha \\ Radio and Spectrum Laboratory, KDDI Research, Inc., 2-1-15 Ohara, Fujimino-shi, Saitama, Japan \\ Correspondence should be addressed to Rony Kumer Saha; rony107976@gmail.com
}

Received 1 January 2021; Revised 20 March 2021; Accepted 16 April 2021; Published 3 May 2021

Academic Editor: Claudia Campolo

Copyright (C) 2021 Rony Kumer Saha. This is an open access article distributed under the Creative Commons Attribution License, which permits unrestricted use, distribution, and reproduction in any medium, provided the original work is properly cited.

\begin{abstract}
Power-domain based dynamic spectrum access (PDSA) techniques are proposed for sharing $28 \mathrm{GHz}$ spectrum of any Mobile Network Operator (MNO) with in-building small cells (SCs) of the other countrywide. By controlling the transmission power of SCs, PDSA techniques explore the traditional interweave access by operating an SC at the maximum transmission power and the underlay access by allowing to operate an SC at a lowered transmission power separately, as well as jointly. Average capacity, spectral efficiency, energy efficiency, cost efficiency, and throughput per SC user equipment (UE) are derived for an arbitrary number of MNOs in a country. By varying the spectrum reuse factor for the millimeter-wave spectrum in each building of SCs, extensive numerical and simulation results and analyses for an illustrative scenario of a country consisting of four MNOs are carried out for the interweave and underlay techniques when applying separately, as well as the hybrid interweave-underlay technique and the static licensed spectrum allocation (SLSA) technique. It is shown that, due to gaining more shared spectra, the hybrid interweave-underlay technique provides the best, whereas the SLSA provides the worst, performances of all techniques in terms of the average capacity, spectral efficiency, energy efficiency, cost efficiency, and throughput per UE of an SC. Moreover, we show that the hybrid interweave-underlay technique, the interweave technique, and the underlay technique, respectively, can satisfy the expected requirements of spectral and energy efficiencies for Sixth-Generation (6G) networks by reusing each MNO's $28 \mathrm{GHz}$ spectrum to SCs of about $33.33 \%, 50 \%$, and $50 \%$ less number of buildings than that required by the SLSA for a spectrum reuse factor of six per building of small cells.
\end{abstract}

\section{Introduction}

1.1. Background. Nowadays, radio spectrum scarcity has become a major issue in mobile communications due to a nondynamic or static allocation of spectrum to Mobile Network Operators (MNOs) to serve a growing user demand for high capacity and data rates. Such static allocations of spectrum cause a large segment of the allocated spectrum idle, resulting in a low utilization. As per [1], the occupancy of spectrum below $3 \mathrm{GHz}$ varies from $15 \%$ to $85 \%$, which reflects as well a considerable variation in the utilization of spectrum in that region.

Recently, cognitive radio (CR) has been considered as a key enabling technology to address this spectrum scarcity issue [2]. In CR, spectrum access is a major function [3], which prevents simultaneous access of any spectrum by primary and secondary user equipments (UEs). In this regard, interweave and underlay are two major spectrum access categories in CR that exploit the power domain (PD) to limit the transmission power of secondary UEs (SUs) to reduce interference to primary UEs (PUs) while ensuring the quality-ofservice [4].

In case of interweave, only the unused spectrum by PUs can be accessed by SUs opportunistically. Though interweave access needs additional spectrum sensing by SUs to find an idle spectrum of PUs, SUs are allowed to transmit at the maximum power. In contrast, in the underlay spectrum access, SUs can simultaneously access the spectrum of PUs subject to satisfying the interference threshold set by PUs. In addressing the cochannel interference (CCI) limit by SUs 
toward PUs, even though underlay suffers from the reduced transmission power of SUs, no spectrum sensing is needed by SUs.

Hence, though both the interweave and underlay have pros and cons when employing separately as aforementioned; the combination of these two spectrum access techniques (also termed as hybrid interweave-underlay technique) can improve spectral and energy efficiencies [4] even further. More specifically, SUs can explore the interweave access when the spectrum of PUs is idle and the underlay access when the spectrum of PUs is busy. This allows SUs to operate at the maximum transmission power during an idle period in contrast to operating at the reduced transmission power when employing only the underlay access all the time.

1.2. Related Work and Problem Statement. A number of research works have already addressed the interweave and underlay spectrum access techniques both separately and jointly. For example, several works have already been carried out in the existing literature on the underlay technique as follows. In [5], an interference management method to share spectrum using the underlay access, and in [6], an investigation on the quality-of-service-aware user selection approach for the CR underlay access, have been addressed. Moreover, a study in [7] and a survey in [8] on resource allocation of underlay CR networks have been carried out. Furthermore, in [9], a technique employing the underlay access for small cells located in buildings in the multiple MNO scenario has been presented.

Similarly, regarding the interweave access, the authors in [10] have investigated the resource allocation in interweaved primary and secondary multicast networks, whereas, in [11], the authors have addressed the stable throughput of an interweave CR. Further, the authors in [12] have provided a detailed analysis of the interweave policy-based CR network. Furthermore, in [13], the authors have presented a novel approach and investigated the impact of imperfect channel knowledge on the performance of the interweave systems, whereas, in [14], the author has presented an interweave shared-use model in millimeter-wave (mmWave) systems.

Moreover, a number of existing researches have addressed already the hybrid or joint interweave-underlay spectrum access technique. For example, the author in [15] has proposed a novel hybrid interweave-underlay spectrum access and reuse technique for the countrywide full $28 \mathrm{GHz}$ spectrum. Moreover, in [16], a hybrid underlay-interweave mode enabled cognitive radio network (CRN) scheme, and in [17], a hybrid interweave-underlay spectrum access scheme in the $5 \mathrm{GHz}$ has been proposed. Besides, for the performance analysis, the downlink capacity region of a secondary network has been presented by the authors in [18] for a hybrid underlay-interweave paradigm. However, a consensus about the more suitable spectrum access out of the interweave and underlay for SUs is not too obvious [2].

Besides, most data are originated indoors, particularly in urban buildings installed with small cells [19]. Hence, it is critical to serve high capacity demand in such buildings. In this regard, operating small cells in the mmWave spectrum bands in such buildings can be a promising candidate. Along this direction, a comparative study relating to the PD based dynamic spectrum access techniques in CRN, particularly, when employing the interweave and the underlay spectrum access techniques separately, as well as jointly, to avail more spectrum within multistory buildings may contribute to serve the growing capacity demand indoors, which, however, has not been addressed in the existing literature.

\subsection{Contribution and Novelty}

(1) Contribution. To address the issues outlined above, PD based dynamic spectrum access (PDSA) techniques are proposed for the dynamic spectrum access of the $28 \mathrm{GHz}$ spectrum of an $\mathrm{MNO}$, referred to as primary MNO ( $\mathrm{p}-\mathrm{MNO}$ ), by in-building small cells of the other, referred to as secondary MNO (s$\mathrm{MNO}$ ) by controlling the transmission power of small cells of each MNO countrywide. The proposed PDSA techniques explore the traditional interweave and underlay spectrum access techniques both separately and jointly. The following are contributed in this paper

(i) The proposed techniques along with the system architecture are presented

(ii) We then derive generic expressions for the average capacity, spectral efficiency (SE), energy efficiency (EE), cost efficiency (CE), and throughput per small cell UE performance metrics for a country with an arbitrary number of MNOs

(iii) Extensive numerical and simulation results and analyses are carried out to evaluate the performance when employing the interweave and underlay spectrum access techniques jointly, as well as separately

(iv) Finally, we evaluate the SE and EE performances of the proposed techniques against the $\mathrm{SE}$ and EE requirements for Sixth-Generation (6G) networks

(2) Novelty. The above contribution is an extended version of the conference work [20]. The novelty of the contribution due to the extension to [20] can be summarized as follows

(a) Relaxation of the Constraint and Estimation of the Generic Performance Metric. In [20], the mathematical analysis and evaluation are carried out for a specific number of MNOs (i.e., four MNOs) in a country. We relax this constraint by considering MNOs of any number countrywide. Correspondingly, generic expressions for the performance metrics are deduced and analyzed by introducing newly the properties of Pascal's triangle and the equal likelihood criterion 
(b) Spectrum Reusing. Unlike [20], we consider exploiting the spectrum allocated to each MNO in the primary level (even after sharing with other MNOs) further in the 3-dimensional space of a multistory building of small cells to reuse the same spectrum of each MNO more than once to its small cells within the building subject to satisfying a minimum CCI threshold set by the operators in prior

(c) Inclusion of New Result and Discussion. Unlike [20] that deals only with the hybrid access technique in $\mathrm{PD}$, we explore the interweave and the underlay access techniques separately, as well as jointly, to provide a complete PD based dynamic spectrum access analysis by presenting their relative outperformance when each of them is employed individually, as well as in a hybrid mode. Moreover, two more performance metrics, including average CE and throughput per small cell UE, are introduced newly, and performance evaluations of the proposed techniques in terms of these metrics are carried out

1.4. Organization. The following are performed section-wise to address PDSA techniques. In Section 2, PDSA techniques and system architectures are discussed. In Section 3, we perform relevant mathematical analysis to derive average capacity, SE, EE, CE, and throughput per small cell UE performance metrics by employing the proposed techniques. Parameters and assumptions, as well as simulation results and analyses are presented in Section 4 to demonstrate that employing interweave and underlay techniques jointly performs better than when employing each of them separately. Moreover, we also show that the interweave and underlay spectrum access techniques, regardless of employing them separately or jointly, can address both SE and EE requirements for $6 \mathrm{G}$ networks. The paper is concluded in Section 5.

\section{System Architecture and Proposed Techniques}

2.1. System Architecture. We consider that an arbitrary number of $O$ MNOs is operating in a country. An example of system architecture is shown in Figure 1 for four MNOs (i.e., $O=4$ ), represented as $\mathrm{MNO} o$, where $o \in\{1,2,3,4\}$. Assume that each MNO has a similar architecture, and hence, only MNO 1 is shown in detail in Figure 1(a) for simplicity. All small cell base stations (SBSs) are deployed only within multistory buildings. Though in practice, an SBS can serve multiple users simultaneously, we limit the maximum number of users that an SBS can serve at a time to one to take advantage of the system-level modeling due to its insignificant variation with the number of users served simultaneously by an SBS. Picocell base stations (PBSs) help offload a portion of traffic of the macrocell base station (MBS). All in-building SBSs operate at the $28 \mathrm{GHz}$, whereas MBSs and PBSs operate at the $2 \mathrm{GHz}$. Each MNO is allocated to the same amount of the $28 \mathrm{GHz}$ spectrum, which can be shared among them by employing the proposed PDSA technique. An illustration of sharing $28 \mathrm{GHz}$ spectra of other MNOs with a small cell of MNO 1 is shown in Figure 1(b).

Remark 1. An SBS usually serves a small group of users within a small area, and the speed of a UE in the indoor environment is very low such that the channel variation at this speed can be considered negligible for a Line-Of-Sight (LOS) $28 \mathrm{GHz}$ mmWave channel. Further, since Resource Blocks (RBs) are allocated orthogonally by any proportional fair scheduler to small cell UEs of an MNO, the overall system-level capacity and other associated performance metrics are not affected by the change in the number of active small cell UEs per SBS (i.e., small cell UE density). Hence, following numerous existing literature $[21,22]$ that considered as well the user density of one UE per small cell (e.g., femtocell), we also adopt that each SBS serves only one UE at a time. Certainly, in practice, the UE density per SBS can be more than one. However, such simplicity in assumptions is suitable for closed-form expressions and reasonable for the system-level performance evaluations.

2.2. Proposed Techniques. We propose power-domain based dynamic spectrum access (PDSA) techniques by controlling the transmission power of in-building small cells of any $\mathrm{MNO}$ in a country stated as follows. The $28 \mathrm{GHz}$ spectrum allocated to an MNO, called primary-MNO ( $p-M N O)$, in a country can be shared with small cells in a building of the other $M N O$, called secondary-MNO (s-MNO), subject to satisfying the condition that each small cell of the s-MNO operates at the maximum transmission power if no UE of the p-MNO is present, whereas at a reduced transmission power if a UE of the $p-M N O$ is present. The transmission power of small cells of the s-MNO is reduced commensurate with the interference threshold set in prior by the $p-M N O$.

As indicated above, using the PDSA techniques, in addition to operating at either the interweave spectrum access technique (i.e., operating at the maximum transmission power) or the underlay spectrum access technique (i.e., operating at a reduced transmission power) separately, by exploring both techniques jointly based on the presence of UEs of p-MNOs, in-building small cells of an s-MNO in a country can be operated in a hybrid interweave-underlay technique.

In executing the proposed PDSA techniques, SBSs of the s-MNO keep sensing to identify UEs of each p-MNO to update the spectrum access mode to either the interweave or the underlay or the hybrid interweave-underlay to limit CCI to UEs of the respective p-MNO. In this regard, to switch between the transmission power levels corresponding to the interweave and underlay spectrum accesses, both reactive (where an s-MNO executes the sensing operation) and proactive (where the arrival of UEs of a p-MNO are predicted based on the knowledge of traffic model [23]) spectrum sensing techniques can be used.

Moreover, since the licensed spectrum of an $\mathrm{MNO}$ can be reused in space subject to satisfying a minimum CCI, we consider employing the spectrum reusing technique on top of the dynamic spectrum access (DSA) techniques by exploiting the space-domain. In this regard, by forming a three- 


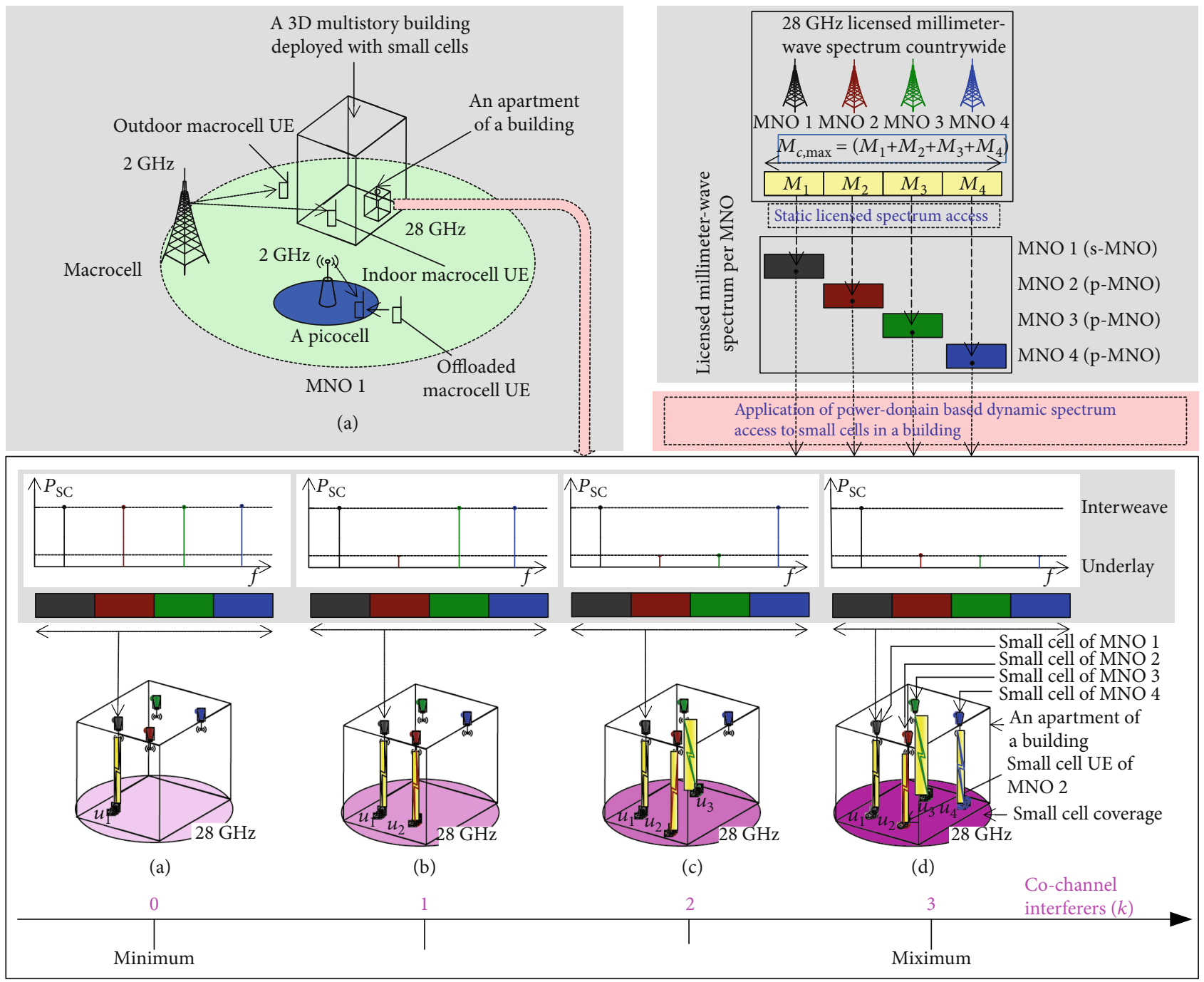

(b)

FIGURE 1: A system architecture consisting of $O=4$ MNOs in a country. $P_{\mathrm{SC}}$ denotes the transmission power of an in-building small cell base station of MNO 1.

dimensional cluster of small cells within a building subject to satisfying a minimum CCI between cochannel SBSs, the same spectrum allocated to each MNO in the primary-level can be reused to improve the spectrum utilization further.

\section{Mathematical Analysis}

3.1. Preliminary. Consider that there are $O$ MNOs countrywide which are allocated to a total of $M_{C \text {, max }} 28 \mathrm{GHz}$ spectrum. $M_{C, \max }$ is expressed in Resource Blocks (RBs), and an $\mathrm{RB}$ is equal to $180 \mathrm{kHz}$. Denote $M_{o}$ as the $28 \mathrm{GHz}$ spectrum allocated to an MNO $o$ such that $o \in \mathbf{O}=\{1,2, \cdots, O\}$ and $\sum_{o=1}^{O} M_{o} \leq M_{C \text {,max }}$. Further, let $S_{M}$ and $S_{P}$ denote the number of macrocells and picocells per macrocell, respectively, of any MNO. Also, let $L$ denote the number of buildings per macrocell and $S_{F}$ denote the number of small cells per building. Assume that each building has $S_{F}$ small cells. Also, denote $\mathbf{T}$ as the simulation run time such that $\mathbf{T}=\{1,2,3, \cdots, Q\}$.
$Q$ denotes the maximum time (in time step each lasting $1 \mathrm{~ms})$.

The transmission power of an MBS and a PBS is denoted, respectively, as $P_{\mathrm{MC}}$ and $P_{\mathrm{PC}}$. Let $P_{\mathrm{SC}, \max , o}$ and $P_{\mathrm{SC}, \text { red, } o}$ denote, respectively, the maximum transmission power and the reduced transmission power of an SBS of MNO $o$ when operating under the interweave and underlay spectrum access techniques such that $P_{\mathrm{SC}, \max , o}>P_{\mathrm{SC}, \mathrm{red}, o}$. Denote $I_{\text {thr,und }}$ as the interference threshold at a UE of a p-MNO in the underlay spectrum access. Then, the transmission power of a small cell of an MNO $o$ needs to be adaptive as follows [24] when operating in the underlay access for an interference channel gain of $\kappa$.

$$
P_{\mathrm{SC}, \text { und }, o}=\left\{\begin{array}{cl}
P_{\mathrm{SC}, \text { red }, o}, & \left(\kappa P_{\mathrm{SC}, \text { red }, o}\right) \leq I_{\text {thr,und }} \\
\left(I_{\text {thr,und }} / \kappa\right), & \left(\kappa P_{\mathrm{SC}, \text { red }, o}\right)>I_{\text {thr,und }}
\end{array}\right\} .
$$


The received Signal-to-Interference-Plus-Noise Ratio (SINR) at $\mathrm{RB}=i$ in Transmission Time Interval $(\mathrm{TTI})=t$ at a UE of an MNO $o$ can be given by

$$
\rho_{t, i, o}=\left(\frac{P_{t, i, o}}{\left(\left(N_{t, i, o}^{s}+I_{t, i, o}\right)\right)}\right) \cdot H_{t, i, o}
$$

where $P_{t, i, o}, N_{t, i, o}^{\mathrm{s}}, I_{t, i, o}$, and $H_{t, i, o}$ denote, respectively, transmission power, noise power, interference power, and link loss at $\mathrm{RB}=i$ in TTI $=t$ of an MNO $o . H_{t, i, o}$ is given by

$$
H_{t, i, o}(\mathrm{~dB})=\left(G_{t}+G_{r}\right)-\left(L_{F}+\mathrm{PL}_{t, i, o}\right)+\left(\mathrm{LS}_{t, i, o}+\mathrm{SS}_{t, i, o}\right)
$$

where $\left(G_{t}+G_{r}\right)$ and $L_{F}$ denote, respectively, total antenna gain and connector loss. Also, $\mathrm{LS}_{t, i, o}$ denotes large-scale fading, $\mathrm{SS}_{t, i, o}$ denotes small-scale fading, and $\mathrm{PL}_{t, i, o}$ denotes path loss between a base station (BS) and a UE of an MNO $o$ at $\mathrm{RB}=i$ in $\mathrm{TTI}=t$.

Denote $\beta$ as the implementation loss factor. Then, a link throughput at $\mathrm{RB}=i$ in TTI $=t$ for an $\mathrm{MNO} o$ in bps per $\mathrm{Hz}$ is given by $[25,26]$.

$$
\sigma_{t, i, o}\left(\rho_{t, i, o}\right)=\left\{\begin{array}{cc}
0, & \rho_{t, i, o}<-10 \mathrm{~dB} \\
\beta \log _{2}\left(1+10^{\left(\rho_{t, i, o}(\mathrm{~dB}) / 10\right)}\right), & -10 \mathrm{~dB} \leq \rho_{t, i, o} \leq 22 \mathrm{~dB} \\
4.4, & \rho_{t, i, o}>22 \mathrm{~dB}
\end{array}\right\} .
$$

Using (4), the total capacity of macrocell UEs of MNO $o$ is given by

$$
\sigma_{\mathrm{MBS}, o}=\sum_{t=1}^{Q} \sum_{i=1}^{M_{\mathrm{MBS}, o}} \sigma_{t, i, o}\left(\rho_{t, i, o}\right)
$$

where $M_{\mathrm{MBS}, o}$ denotes $2 \mathrm{GHz}$ spectrum in RBs of an MBS of MNO o. $\sigma$ and $\rho$ are estimated over $M_{\mathrm{MBS}, o} \mathrm{RBs}$ in $t \in \boldsymbol{T}$ for MNO $o$.

3.2. Spectrum Sharing in PD. Recall that we consider one small cell per MNO $o$, which can serve a maximum of one $\mathrm{UE}$ at a time in each apartment of a building. A UE of an MNO o may either exist (represented by binary 1) or not (represented by binary 0 ) in an apartment such that $O$ UEs can coexist in a maximum of $2^{O}$ possible ways, as shown in Table 1 for $O=4$. Let each combination of coexistence of $O$ UEs in an apartment is equally likely. Hence, given the existence (i.e., the binary state 1) of a UE of an MNO $o$ over $|\boldsymbol{T}|$ $=Q$, UEs of other MNOs $\boldsymbol{O} \backslash o$ can coexist with that of $\mathrm{MNO} o$ in a maximum of $2^{O-1}$ (i.e., 8 for $O=4$ ) possible ways (Table 1$)$; each occurs with a probability of $2^{-(O-1)}$ (i.e., $1 / 8$ for $\mathrm{O}=4)$.

For example, as given in Table 1 , for a UE $u_{1}$ of MNO 1 as an s-MNO, all the possible combinations in which $u_{1}$ can coexist with other UEs $u_{2}, u_{3}$, and $u_{4}$ of MNO 2, MNO 3, and MNO 4 as p-MNOs, respectively, are the following $\left\{u_{1}\right\}$

\begin{tabular}{|c|c|c|c|c|c|c|}
\hline \multirow{2}{*}{$u_{1}$} & \multirow{2}{*}{$u_{2}$} & \multirow{2}{*}{$u_{3}$} & \multirow{2}{*}{$u_{4}$} & \multicolumn{2}{|c|}{$\begin{array}{l}\text { Shared spectrum for } \\
\qquad u_{1}\end{array}$} & \multirow{2}{*}{$\begin{array}{c}\text { Licensed spectrum for } u_{1} \\
\text { Both interweave and } \\
\text { underlay }\end{array}$} \\
\hline & & & & Interweave & Underlay & \\
\hline 0 & 0 & 0 & 0 & \multirow{8}{*}{\multicolumn{2}{|c|}{ Not applicable due t }} & \\
\hline 0 & 0 & 0 & 1 & & & \\
\hline 0 & 0 & 1 & 0 & & & \\
\hline 0 & 0 & 1 & 1 & & & $o$ the nonexistence of $u_{1}$ \\
\hline 0 & 1 & 0 & 0 & & & \\
\hline 0 & 1 & 0 & 1 & & & \\
\hline 0 & 1 & 1 & 0 & & & \\
\hline 0 & 1 & 1 & 1 & & & \\
\hline 1 & 0 & 0 & 0 & $3 M$ & 0 & $M$ \\
\hline 1 & 0 & 0 & 1 & $2 M$ & $M$ & $M$ \\
\hline 1 & 0 & 1 & 0 & $2 M$ & $M$ & $M$ \\
\hline 1 & 0 & 1 & 1 & $M$ & $2 M$ & $M$ \\
\hline 1 & 1 & 0 & 0 & $2 M$ & $M$ & $M$ \\
\hline 1 & 1 & 0 & 1 & $M$ & $2 M$ & $M$ \\
\hline 1 & 1 & 1 & 0 & $M$ & $2 M$ & $M$ \\
\hline 1 & 1 & 1 & 1 & 0 & $3 M$ & $M$ \\
\hline
\end{tabular}
, $\left\{u_{1}, u_{2}\right\},\left\{u_{1}, u_{3}\right\},\left\{u_{1}, u_{4}\right\},\left\{u_{1}, u_{2}, u_{3}\right\},\left\{u_{1}, u_{2}, u_{4}\right\},\left\{u_{1}\right.$,
TABLE 1: Coexistence and shared spectrum for UE $\mathrm{u}_{1}$ of MNO 1 using the proposed technique.

$\left.u_{3}, u_{4}\right\}$, and $\left\{u_{1}, u_{2}, u_{3}, u_{4}\right\}$. Recall that each MNO is allocated to the same amount of the $28 \mathrm{GHz}$ spectrum of $M$ RBs such that the above possible combinations correspond to the amount of shared spectrum for $u_{1}$ of $3 M, 2 M, 2 M, 2 M, M$, $M, M$, and 0 , respectively, for the interweave access, and of $0, M, M, M, 2 M, 2 M, 2 M$, and $3 M$ for the underlay access, as shown in Table 1. These correspond to the total spectrum for $u_{1}$ of $4 M, 3 M, 3 M, 3 M, 2 M, 2 M, 2 M$, and $M$, respectively, for the interweave access, and of $M, 2 M, 2 M, 2 M, 3 M, 3 M$, $3 M$, and $4 M$ for the underlay access.

Hence, using Table 1, for an arbitrary number of MNOs $O$ countrywide, the components of the shared spectra can be expressed as $\{0, M, 2 M, \cdots,(n-1) M, n M\}$ where $n=(O$ - 1) such that Table 1 follows a left-justified Pascal's triangle [27] as shown in Table 2 for $O=4$. Each entry in row $n$ and column $k$, where $n \geq k \geq 0$, in Table 2 implies a binomial coefficient $\mathbf{C}(n, k)=\left(\begin{array}{l}n \\ k\end{array}\right)$, which is as a multiplier corresponding to the respective component of the shared spectra $\{0, M, 2 M, \cdots,(n-1) M, n M\}$ for a UE of MNO $o$ in row $n$ of the left-justified Pascal's triangle [27].

Note that the same component of the shared spectrum exists more than once, and the complete set of components of the shared spectrum, where each component of the shared spectrum is scaled by its corresponding multiplier, for a UE of an MNO $o$ (shown in Table 1) can be expressed in Table 2 as follows [27] $\{(\mathbf{C}(n, 0) \times 0),(\mathbf{C}(n, 1) \times M),(\mathbf{C}(n$, 2) $\times 2 M), \cdots,(\mathbf{C}(n, n) \times n M)\}$.

Assume that $\rho_{t, i, o, \text { int }}$ and $\rho_{t, i, o, \text { und }}$ denote, respectively, the received SINR at RB $=i$ in TTI $=t$ at a UE of an MNO $o$ when 
TAble 2: Left-justified Pascal's triangle for $O=4$ to define a multiplier corresponding to the respective shared spectrum components in Table 1 [27].

\begin{tabular}{|c|c|c|c|c|c|c|}
\hline \multirow[t]{2}{*}{$O$} & \multirow[t]{2}{*}{$n=O-1$} & \multicolumn{4}{|c|}{$\begin{array}{l}\text { Multiplication entry for the } \\
\text { components of the shared } \\
\text { spectra }\end{array}$} & \multirow[t]{2}{*}{$2^{n}$} \\
\hline & & 0 & $M$ & $2 M$ & $3 M$ & \\
\hline 1 & 0 & 1 & & & & 1 \\
\hline 2 & 1 & 1 & 1 & & & 2 \\
\hline 3 & 2 & 1 & 2 & 1 & & 4 \\
\hline 4 & 3 & 1 & 3 & 3 & 1 & 8 \\
\hline
\end{tabular}

operating under the interweave spectrum access and the underlay spectrum access techniques. Let $\sigma_{t, i, o, \text { int }}$ and $\sigma_{t, i, o \text {,und }}$ denote, respectively, the link throughput corresponding to $\rho_{t, i, o, \text { int }}$ and $\rho_{t, i, o, \text { und }}$.

Now, using (2) and (4) and following Table 1, the aggregate capacity of $\mathrm{MNO} o$ when employing only the interweave access is given for an SBS $s$, as well as all SBSs $S_{F}$ per building, as follows.

$$
\begin{gathered}
\sigma_{o, s}^{\mathrm{iw}}=\sum_{t=1}^{\left(\mathrm{Q} / 2^{O-1}\right.} \sum_{i=1}^{M} \sigma_{o, t, i}^{\mathrm{iw}}\left(\rho_{o, t, i}^{\mathrm{iw}}\right) \\
+\sum_{k=1}^{O-1}\left(\begin{array}{c}
O-1 \\
k
\end{array}\right)\left(\sum_{t=1}^{\left(\mathrm{Q} / 2^{O-1}\right.} \sum_{i=1}^{k M} \sigma_{o, k, t, i}^{\mathrm{iw}}\left(\rho_{o, k, t, i}^{\mathrm{iw}}\right)\right), \\
\sigma_{o, S_{F}}^{\mathrm{iw}}=\sum_{s=1}^{S_{F}} \sigma_{o, s}^{\mathrm{iw}} .
\end{gathered}
$$

Similarly, when employing only the underlay spectrum access technique, the aggregate capacity of MNO $o$ is given for a small cell $s$, as well as all small cells $S_{F}$ per building, as follows.

$$
\begin{gathered}
\sigma_{o, s}^{\mathrm{ul}}=\sum_{t=1}^{\left(\mathrm{Q} / 2^{O-1}\right.} \sum_{i=1}^{M} \sigma_{o, t, i}^{\mathrm{iw}}\left(\rho_{o, t, i}^{\mathrm{iw}}\right) \\
+\sum_{k=1}^{O-1}\left(\begin{array}{c}
O-1 \\
k
\end{array}\right)\left(\sum_{t=1}^{\left(\mathrm{Q} / 2^{O-1}\right.} \sum_{i=1}^{k M} \sigma_{o, k, t, i}^{\mathrm{ul}}\left(\rho_{o, k, t, i}^{\mathrm{ul}}\right)\right), \\
\sigma_{o, S_{F}}^{\mathrm{ul}}=\sum_{s=1}^{S_{F}} \sigma_{o, s}^{\mathrm{ul}} .
\end{gathered}
$$

However, when employing the hybrid interweaveunderlay spectrum access technique, the aggregate capacity of MNO $o$ can be given for a small cell $s$, as well as all small cell $S_{F}$ per building, as follows.

$$
\begin{aligned}
& \sigma_{o, s}^{\mathrm{hy}}= \sum_{t=1}^{Q} \sum_{i=1}^{M} \sigma_{o, t, i}^{\mathrm{iw}}\left(\rho_{o, t, i}^{\mathrm{iw}}\right) \\
&+\sum_{k=1}^{O-1}\left(\begin{array}{c}
O-1 \\
k
\end{array}\right)\left(\sum_{t=1}^{\left(\mathrm{Q} / 2^{O-1}\right)} \sum_{i=1}^{k M}\left(\begin{array}{c}
\sigma_{o, k, t, i}^{\mathrm{iw}}\left(\rho_{o, k, t, i}^{\mathrm{iw}}\right)+ \\
\sigma_{o, k, t, i}^{\mathrm{ul}}\left(\rho_{o, k, t, i}^{\mathrm{ul}}\right)
\end{array}\right)\right) \\
& \sigma_{o, S_{F}}^{\mathrm{hy}}=\sum_{s=1}^{S_{F}} \sigma_{o, s}^{\mathrm{hy}} .
\end{aligned}
$$

Hence, for (11), the countrywide aggregate capacity of all MNOs $O$ for a building of SBSs is given by,

$$
\sigma_{O, S_{F}}^{\mathrm{hy}}=\sum_{o=1}^{O} \sum_{s=1}^{S_{F}} \sigma_{o, s}^{\mathrm{hy}}
$$

Like (12), using (6) and (8), we can derive the countrywide capacity when employing the interweave spectrum access technique and the underlay spectrum access technique, respectively. Hence, in what follows, for brevity, we consider only the hybrid interweave-underlay spectrum access for further analysis. However, the analysis is equally applicable when employing the interweave, as well as the underlay, spectrum access techniques.

3.3. Spectrum Reusing. The spectrum allocated to each MNO $o$ in the primary level even after sharing with other MNOs can be exploited further in the 3D space of a multistory building of small cells. A minimum distance between cochannel small cells located on the same floor and across different floors for reusing the same $28 \mathrm{GHz}$ spectrum multiple times within a multistory building of small cells can be defined subject to satisfying a minimum CCI threshold set by the operator in prior. The number of small cells that falls under the 3D space within the building represents the size of a 3D cluster of small cells such that the same spectrum can be reused for MNO $o$ to each cluster of small cells.

Moreover, the external wall penetration loss for a building is high, particularly for a high-frequency signal such that reusing the same spectrum can be reused even to the adjacent building of small cells. Hence, by varying the spectrum reuse factor (RF) both vertically within the same building and horizontally between adjacent buildings, the required capacity can be obtained. Following [28], a minimum distance between cochannel small cells for the $28 \mathrm{GHz}$ mmWave spectrum in the intrafloor level and interfloor level, respectively, for $\mathrm{MNO} o$ can be given by,

$$
\Delta_{a}=\Delta_{m} \times\left(\frac{\Xi_{a}}{I_{a}^{\mathrm{thr}}}\right)^{(1 / 1.797)},
$$

$$
\Delta_{e} \geq \Delta_{m} \times\left(\frac{\left(\Xi_{e} / I_{e}^{\mathrm{thr}}\right)}{10^{\left(\alpha_{f}\left(\Delta_{e}\right) / 10\right)}}\right)^{(1 / 1.797)}
$$


where $I_{a}^{\text {thr }}$ and $I_{e}^{\text {thr }}$ denote, respectively, intrafloor and interfloor CCI constraints at a small cell UE. $\Xi_{a}$ and $\Xi_{e}$ denote, respectively, the maximum number of cochannel small cells in the intrafloor level and interfloor level. $\Delta_{m}$ denotes the minimum distance between a cochannel small cell and a small cell UE, and $\alpha_{f}\left(\Delta_{e}\right)$ denotes the floor penetration loss at the $28 \mathrm{GHz}$ mmWave spectrum.

Let $s_{l}^{a}$ and $s_{l}^{e}$ denote, respectively, the number of small cells corresponding to $\Delta_{a, l}$ and $\Delta_{e, l}$ in a building $l$ such that a 3D cluster consists of $S_{3 \mathrm{D}, l}=\left(s_{l}^{a} \times s_{l}^{e}\right)$ small cells. Hence, the same spectrum of MNO $o$ can be reused for each cluster of $\left(s_{l}^{a} \times s_{l}^{e}\right)$ small cells in a building. Let $S_{F, l}$ denote the maximum number of small cells of MNO $o$ in a building $l$ such that the number of times the same spectrum of MNO $o$ can be reused in building $l$ (i.e., the spectrum RF for MNO $o$ in building $l$ ) is given by

$$
\omega_{l}=\frac{S_{F, l}}{S_{3 \mathrm{D}, l}} .
$$

Hence, for $L$ number of buildings of small cells in a macrocell of MNO $o$, the spectrum RF for a macrocell of $\mathrm{MNO} o$ is given by,

$$
\omega_{\mathrm{MC}}=\sum_{l=1}^{L} \omega_{l}
$$

Because of low multipath fading effect and hence less channel variation of high-frequency signals indoors, we assume a similar channel condition for all buildings of small cells. Then, for the same number of SBSs per building (i.e., $\left.\forall l S_{F, l}=S_{F}\right)$ such that $\forall l \omega_{l}=\omega$ for each MNO $o$, the spectrum $\mathrm{RF}$ for a macrocell of MNO $o$ is given by,

$$
\omega_{\mathrm{MC}}=(L \times \omega) .
$$

3.4. System-Level Performance Metrics. Using linear approximation and considering $\forall l S_{F, l}=S_{F}$ and $\forall l \omega_{l}=\omega$ for MNO $o$, the countrywide system-level average capacity and SE of all MNOs $O$ can be given, respectively, for $L>1$ as follows.

$$
\begin{aligned}
& \sigma_{O}^{\text {hy,CP }}=\sum_{o=1}^{O}\left(\sigma_{\mathrm{MBS}, o}+\sum_{l=1}^{L}\left(\omega_{l} \times \sum_{s=1}^{S_{3 \mathrm{D}, l}} \sigma_{o, s}^{\mathrm{hy}}\right)\right), \\
& \sigma_{O}^{\mathrm{hy}, \mathrm{SE}}=\frac{\sigma_{O}^{\mathrm{hy}, \mathrm{CP}}}{\left(\left(M_{\mathrm{C}, \max }+\sum_{o=1}^{O} M_{\mathrm{MBS}, o}\right) \times Q\right)} .
\end{aligned}
$$

Since each SBS can serve one UE at any time, the average throughput per small cell UE of MNO $o$ in building $l$ can be expressed as follows.

$$
\sigma_{o}^{\text {hy,thr }}=\frac{\left(\omega_{l} \times \sum_{s=1}^{S_{3 \mathrm{D}, l}} \sigma_{o, s}^{\mathrm{hy}}\right)}{S_{F}} .
$$

Recall that, in PD, the transmission power of an SBS of MNO $o$ varies with the existence of UEs of other MNOs $\mathbf{O}$ $\backslash$. We consider a separate transceiver for the spectrum of each MNO o of each SBS to switch on and off easily to save the transmission power. Hence, there are $O$ transceivers per SBS of MNO $o$ where one transceiver is operating at the $28 \mathrm{GHz}$ spectrum of $\mathrm{MNO} o$ itself and the remaining $(O-1$ ) transceivers are operating at the shared spectrum of other MNOs $\mathbf{O} \backslash o$ such that the average EE in Joules/bit in PD for the hybrid interweave-underlay spectrum access can be given by,

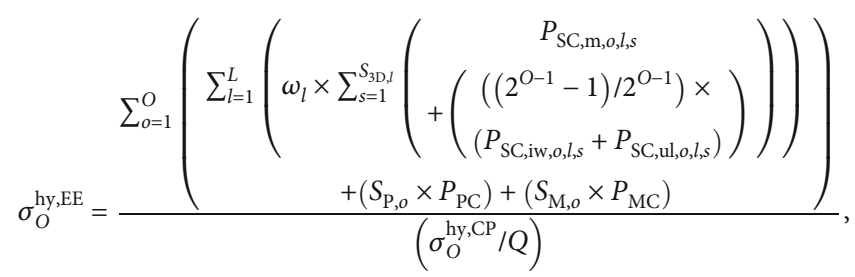

where $P_{\mathrm{SC}, \mathrm{m}, o, l, s}, P_{\mathrm{SC}, \mathrm{iw}, o, l, s}$, and $P_{\mathrm{SC}, \mathrm{ul}, o, l, s}$ denote, respectively, the transmission power of a small cell $s$ of MNO $o$ in a building $l$ when operating at the maximum transmission power, the transmission power with employing the interweave spectrum access, and the transmission power with employing the underlay spectrum access such that $P_{\mathrm{SC}, \mathrm{iw}, o, l, \mathrm{~s}}=P_{\mathrm{SC}, \mathrm{m}, \mathrm{o}, l, \mathrm{~s}}$ and $P_{\mathrm{SC}, \mathrm{ul}, o, l, s}<P_{\mathrm{SC}, \mathrm{m}, o, l, s}$. Similarly, following (21), the average $\mathrm{EE}$ in $\mathrm{PD}$ when employing either the interweave spectrum access or the underlay spectrum access can be expressed.

Now, for the cost efficiency (CE), which can be defined as the amount of cost required to achieve per unit achievable average capacity (i.e., per bps), let $\delta_{o}$ denote the cost of the $28 \mathrm{GHz}$ licensed spectrum for an MNO $o$ such that the total cost of the countrywide full-spectrum is given by $\delta_{F}=\sum_{o=1}^{O}$ $\delta_{o}$. Then, the CE for all MNOs $O$ can be expressed as follows.

$$
\sigma_{O}^{\text {hy,CE }}=\frac{\delta_{F}}{\sigma_{O}^{\text {hy,CP }}} .
$$

3.5. SLSA Technique and Improvement Factor. In a static licensed spectrum allocation (SLSA) technique, an equal amount of spectrum of $M$ RBs is allocated exclusively to each MNO. Then, the countrywide system-level average capacity, SE, EE, and CE for all MNOs $O$ when employing the SLSA technique are given, respectively, by,

$$
\sigma_{\mathrm{SLSA}, O}^{\mathrm{CP}}=\sum_{o=1}^{O}\left(\sum_{l=1}^{L}\left(\omega_{l} \times \sum_{s=1}^{S_{3 \mathrm{D}, l}} \sum_{t \in \boldsymbol{T}} \sum_{i=1}^{M} \sigma_{o, l, s, t, i}\left(\rho_{o, l, s, t, i}\right)\right)\right),
$$

$$
\sigma_{\mathrm{SLSA}, O}^{\mathrm{SE}}=\frac{\sigma_{\mathrm{SLSA}, O}^{\mathrm{CP}}}{\left(\left(M_{\mathrm{C}, \max }+\sum_{o=1}^{O} M_{\mathrm{MBS}, o}\right) \times Q\right)},
$$




$$
\begin{gathered}
\sigma_{\mathrm{SLSA}, O}^{\mathrm{EE}}=\frac{\sum_{o=1}^{O}\left(\begin{array}{c}
\sum_{l=1}^{L}\left(\omega_{l} \times \sum_{s=1}^{S_{\mathrm{B}, l}} P_{\mathrm{SC}, \mathrm{m}, o, l, s}\right)+ \\
\left(S_{P, o} \times P_{\mathrm{PC}}\right)+\left(S_{M, o} \times P_{\mathrm{MC}}\right)
\end{array}\right)}{\left(\sigma_{\mathrm{SLSA}, O}^{\mathrm{CP}} / Q\right)}, \\
\sigma_{\mathrm{SLSA}, O}^{\mathrm{CE}}=\frac{\delta_{F}}{\sigma_{\mathrm{SLSA}, O}^{\mathrm{CP}}} .
\end{gathered}
$$

Likewise, the average throughput per small cell UE due to applying SLSA for MNO $o$ in building $l$ is given by,

$$
\sigma_{\mathrm{SLSA}, o}^{\mathrm{thr}}=\frac{\left(\omega_{l} \times \sum_{s=1}^{S_{3 \mathrm{D}, l}} \sum_{t \in T} \sum_{i=1}^{M} \sigma_{o, l, s, t, i}\left(\rho_{o, l, s, t, i}\right)\right)}{S_{F}} .
$$

Hence, the average capacity, SE, EE, CE, and throughput per small cell UE performance improvement factors due to applying the hybrid interweave-underlay spectrum access in comparison with that of the SLSA are given, respectively, by

$$
\begin{gathered}
\sigma_{\mathrm{IF}}^{\mathrm{hy}, \mathrm{CP}}=\frac{\sigma_{O}^{\mathrm{hy}, \mathrm{CP}}}{\sigma_{\mathrm{SLSA}, O}^{\mathrm{CP}},}, \\
\sigma_{\mathrm{IF}}^{\mathrm{hy}, \mathrm{SE}}=\frac{\sigma_{O}^{\mathrm{hy}, \mathrm{SE}}}{\sigma_{\mathrm{SLSA}, O}^{\mathrm{SE}},}, \\
\sigma_{\mathrm{IF}}^{\mathrm{hy}, \mathrm{EE}}=\frac{\sigma_{O}^{\mathrm{hy}, \mathrm{EE}}}{\sigma_{\mathrm{SLSA}, O}^{\mathrm{EE}}}, \\
\sigma_{\mathrm{IF}}^{\mathrm{hy}, \mathrm{CE}}=\frac{\sigma_{O}^{\mathrm{hy}, \mathrm{CE}}}{\sigma_{\mathrm{SLSA}, O}^{\mathrm{CE}}}, \\
\sigma_{\mathrm{IF}}^{\mathrm{hy}, \mathrm{thr}}=\frac{\sigma_{O}^{\mathrm{hy}, \mathrm{thr}}}{\sigma_{\mathrm{SLSA}, O}^{\mathrm{thr}}} .
\end{gathered}
$$

\section{Performance Result and Analysis}

4.1. Default Parameter and Assumption. Table 3 shows selected simulation parameters and assumptions used for evaluating the performance of the proposed PDSA for all MNOs $O$ countrywide. Detailed channel models and antenna characteristics of all types of base stations and UEs can be found in [29-33]. Previously in [20], though the performance evaluation is carried out for four MNOs in a country (i.e., $O=4$ ) as an example scenario, we relax this constraint in this paper by considering an arbitrary number of MNOs in a country. Correspondingly, generic expressions for the performance metrics are derived and analyzed using the properties of Pascal's triangle and the equal likelihood criterion. The randomness in mobile traffic and user characteristics is captured using the equal likelihood criterion to model the presence of UEs of MNOs within each apartment (Table 1). Besides, to operate in the underlay spectrum access, we limit the transmission power of a small cell to $20 \%$ of its maximum value.

\subsection{Performance Result}

(1) Performance Metric Improvement Factor. Improvement in average capacity, SE, EE, and CE, as well as throughput per small cell UE, performances for four MNOs countrywide is shown in Figure 2 due to applying the hybrid interweave-underlay, as well as when applying either the interweave or the underlay spectrum access techniques. It can be found that the hybrid technique improves all these performance metrics considerably as compared to that of the interweave and underlay techniques when applied separately. This is because, using Table 1 , the maximum shared spectrum achieved due to applying the hybrid interweave-underlay technique is 3 times (interweave and underlay techniques each contributing 1.5 times) the licensed $28 \mathrm{GHz}$ spectrum of any MNO of $M$ RBs (e.g., MNO 1 in Table 1) for time $Q$. This causes the hybrid technique to increase the licensed spectrum of $M$ RBs to $4 M$ RBs for each $M N O$ in time $Q$.

Since the capacity and hence the SE, as well as the throughput per small cell UE, are directly proportional to the available spectrum, the hybrid interweave-underlay technique improves the average capacity and SE, as well as throughput per small cell UE, by about 2.9 times with respect to that of SLSA as shown in Figure 2. These correspond to the average capacity, SE, and throughput per small cell UE improvement by about 1.49 times and 1.4 times, respectively, when applying the interweave and underlay techniques separately.

In contrast, since the $\mathrm{EE}$ and $\mathrm{CE}$ are inversely proportional to the capacity [35], i.e., the available spectrum, the hybrid interweave-underlay technique improves the EE by about $73 \%$ and the CE by about $74.4 \%$ with respect to that of SLSA (Figure 2). These correspond to the EE improvement by about $57.46 \%$ and $57.65 \%$, respectively, and the CE improvement by about $60 \%$ and $58.4 \%$, respectively, when applying the interweave and the underlay techniques separately (Figure 2). Note that a slight variation in the EE and $\mathrm{CE}$ responses occurs due to the fact that in addition to the capacity, unlike the CE, the EE depends as well on the transmission power of BSs.

(2) Impact of the Spectrum Reuse Factor. Figures 3(a)3(d) show, respectively, the average capacity, SE, $\mathrm{EE}$, and $\mathrm{CE}$ responses for all four MNOs countrywide by varying the spectrum reuse factor $\omega$ for the mmWave spectrum in each building of small cells due to applying the hybrid interweave-underlay technique, as well as interweave, underlay, and SLSA techniques. Overall, the hybrid interweave-underlay technique provides the best, whereas the SLSA provides the worst, performances of all techniques in terms of the average capacity, SE, EE, and CE

More specifically, with an increase in $L$, a linear increase in the average capacity and SE, whereas a negativeexponential improvement in the EE and CE, are observed for all techniques for any $\omega$. This implies that, unlike the average capacity and SE, the improvement in both EE and 
TABLE 3: Default parameters and assumptions.

\begin{tabular}{|c|c|}
\hline Parameters and assumptions & Value \\
\hline Total number of MNOs in a country & 4 \\
\hline Frequency bands ${ }^{2,5,6}$ & $2 \mathrm{GHz}$ (non-LOS) for MBSs and PBSs, $28 \mathrm{GHz}$ (LOS) for SBSs \\
\hline Spectrum bandwidth per MNO & $50 \mathrm{MHz}(28 \mathrm{GHz}$ band $)$ and $10 \mathrm{MHz}(2 \mathrm{GHz}$ band $)$ \\
\hline Number of MBSs, PBSs, and SBSs per MNO & 1,2 , and 8 (per building) \\
\hline Transmission power ${ }^{1}(\mathrm{dBm})$ and direction & $\begin{array}{c}46 \text { for } \mathrm{MBSs}^{1,4}, 37 \text { for } \mathrm{PBSs}^{1}, 19 \text { for SBSs } \\
\text { interweave access), and } 12.01 \text { for SBSs (when operating in } \\
\text { the underlay access), downlink }\end{array}$ \\
\hline Macrocell UEs & 30 (total) and 2/15(offloaded) \\
\hline Building and small cell models & $\begin{array}{l}L \text { buildings per macrocell, } 2 \text { floors per building, } 4 \text { apartments per floor, } \\
1 \text { small cell per apartment, and } 10 \times 10 \mathrm{~m}^{2} \text { area per apartment }\end{array}$ \\
\hline Scheduler and traffic model ${ }^{2}$ & Proportional fair (frequency domain) and full buffer \\
\hline Type of small cells & Femtocells (closed subscriber group) \\
\hline TTI $^{1}$, simulation run time, scheduler time constant ${ }^{6}$ & $1 \mathrm{~ms}, 8 \mathrm{~ms}$, and $100 \mathrm{~ms}$ \\
\hline
\end{tabular}

Taken ${ }^{1}$ from [29], ${ }^{2}$ from [30], ${ }^{3}$ from [31], ${ }^{4}$ from [32], from ${ }^{5}[33]$, from ${ }^{6}[34]$.

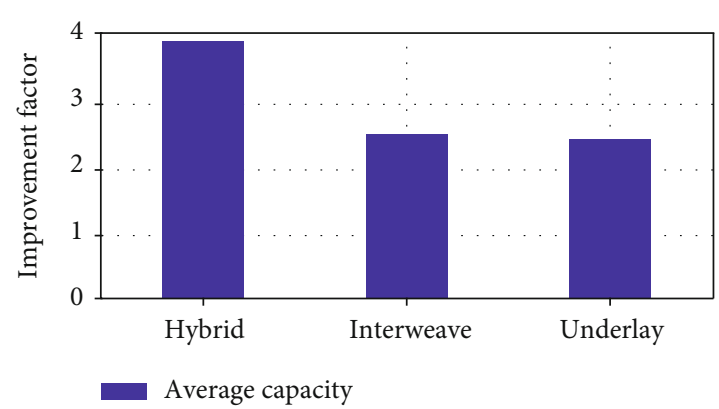

(a)

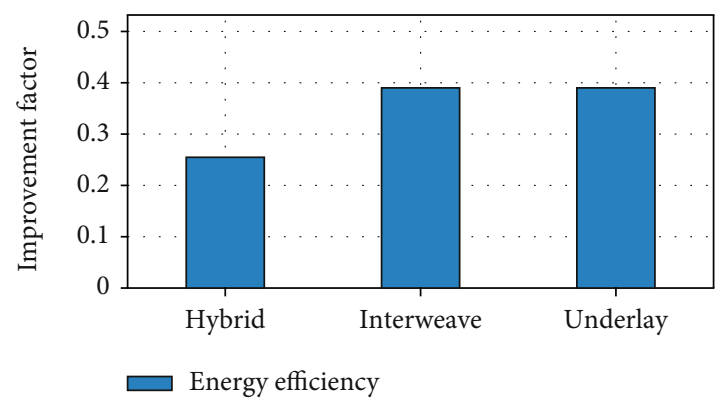

(c)

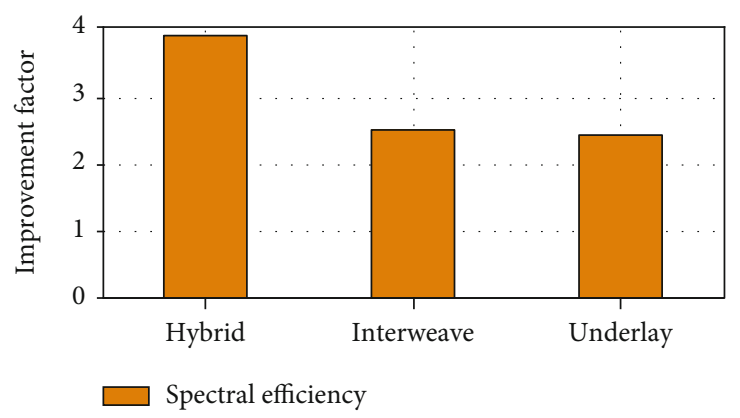

(b)

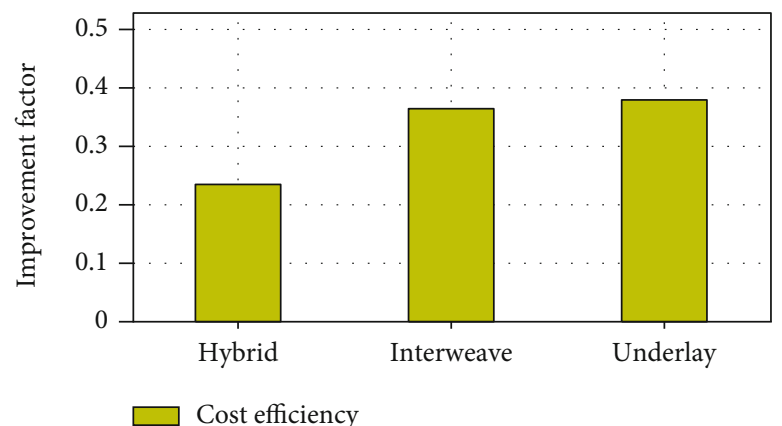

(d)

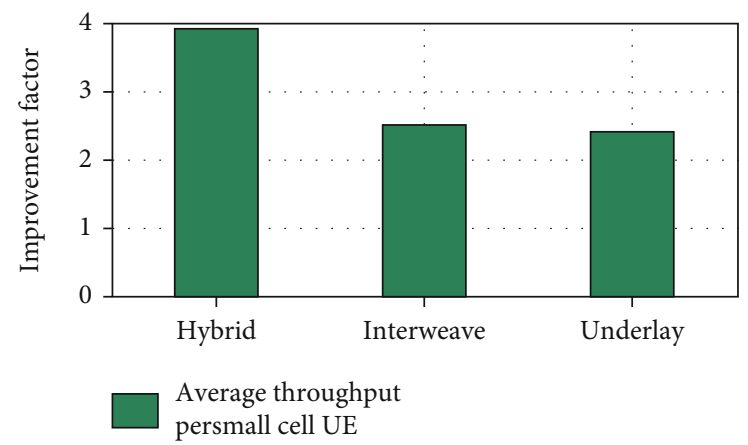

(e)

Figure 2: (a) Average capacity, (b) SE, (c) EE, (d) CE, and (e) throughput per small cell UE improvement factors for four MNOs countrywide due to applying different PD DSA techniques to a single building of SBSs for $\omega$ equals to 1. 


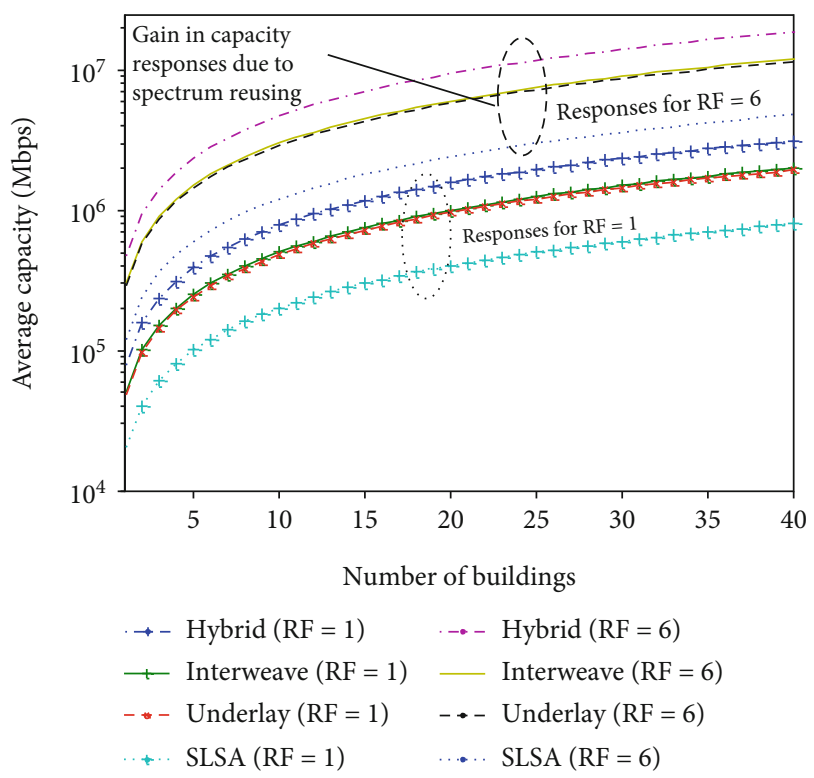

(a)

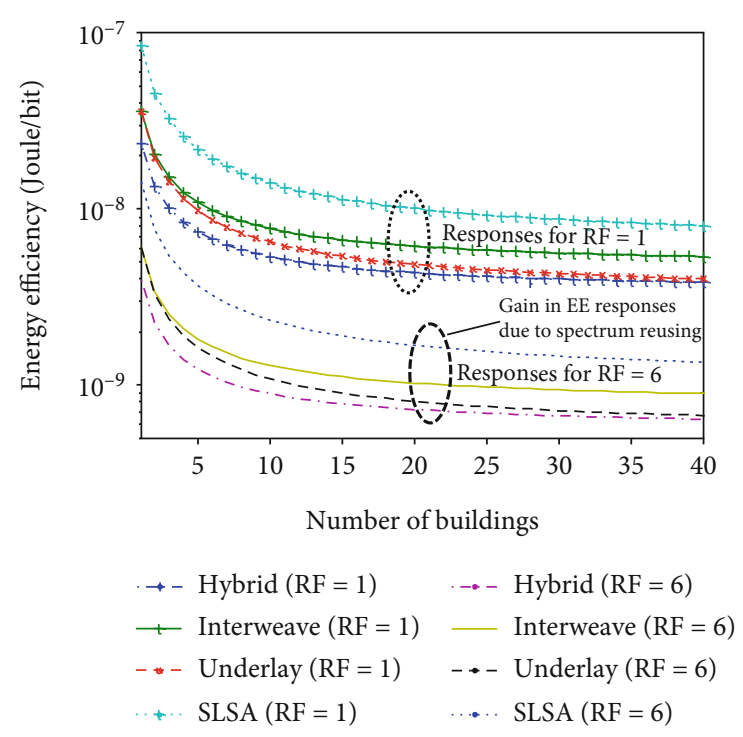

(c)

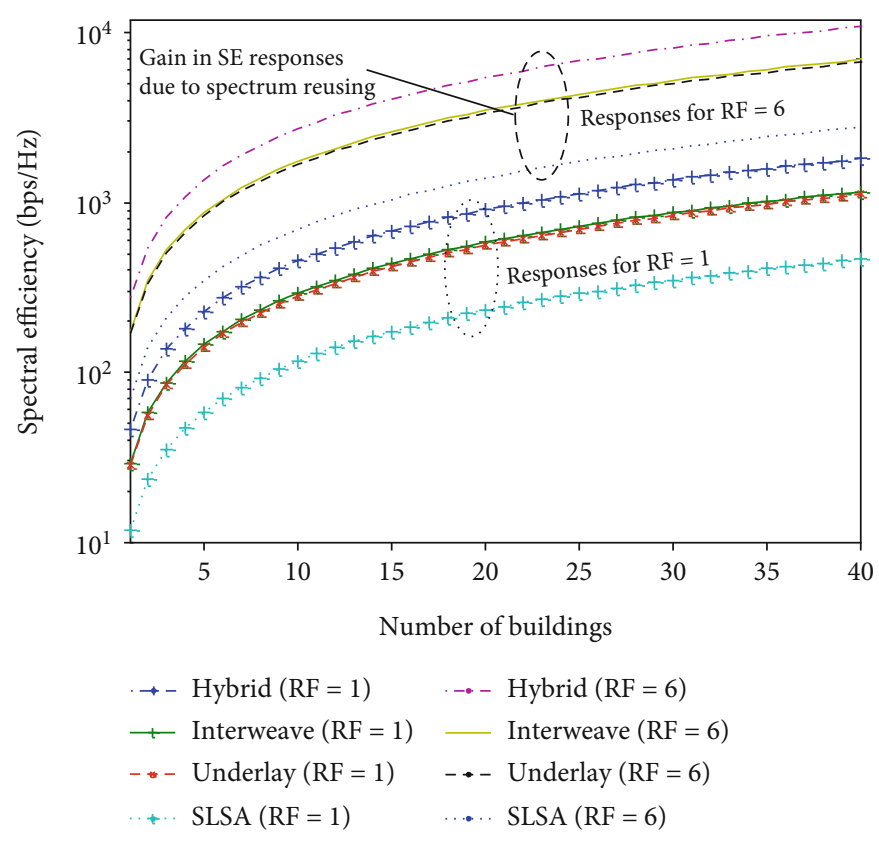

(b)

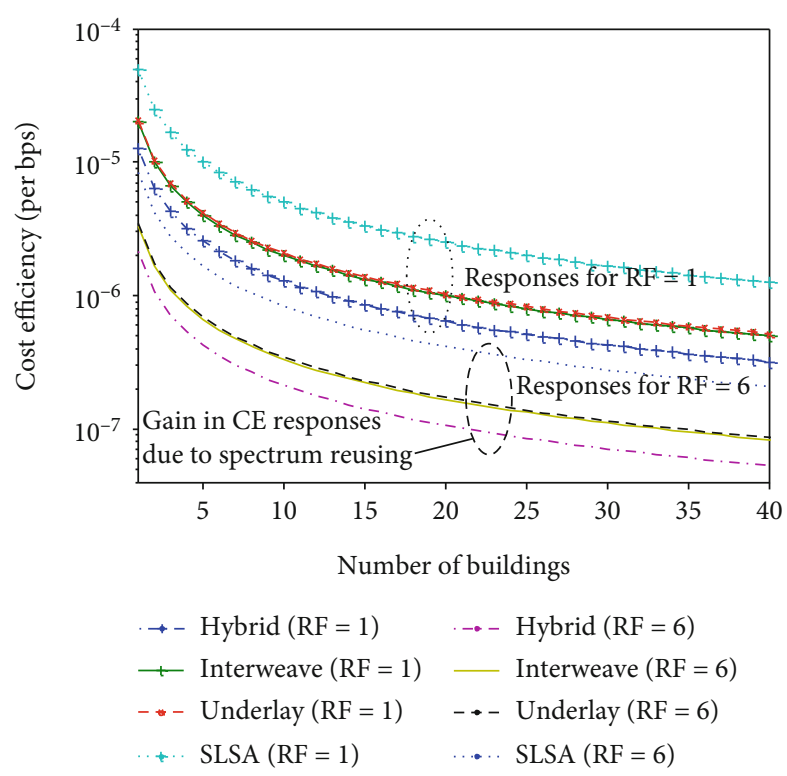

(d)

FIgure 3: (a) Average capacity, (b) SE, (c) EE, and (d) CE performances of four MNOs countrywide due to applying different PD DSA techniques to multiple buildings of SBSs for $\omega$ equals to 1 and 6 .

CE gets to a steady-state value as $L$ tends to a very large value. Moreover, if $\omega$ increases (e.g., from 1 to 6 as shown in Figure 3), all these performance metrics also improve following the same trend as that when $L$ increases. Hence, the overall improvement in any performance metrics is defined by the product of $\omega$ and $L$ such that by varying either $\omega$ or $L$, or both $\omega$ and $L$, the required value of the average capacity, $\mathrm{SE}, \mathrm{EE}$, and CE can be defined. We discuss further the impact of $\omega$ and $L$ with specific examples in the following section.

4.3. Performance Comparison. According to [36, 37], the future $6 \mathrm{G}$ mobile network is expected to provide 10 times average spectral and energy efficiencies of $5 \mathrm{G}$ mobile systems $[38,39]$. In other words, $6 \mathrm{G}$ requires an average SE of 270$370 \mathrm{bps} / \mathrm{Hz}$, as well as average $\mathrm{EE}$ of $0.3 \mu \mathrm{J} / \mathrm{bit}$. Using Figure 3, for $\omega=6$, it can be found that the values of $L$ that can achieve the required spectral and energy efficiencies for $6 \mathrm{G}$ as aforementioned are 2, 3, 3, and 6, respectively, for the hybrid interweave-underlay, interweave, underlay, and SLSA techniques. Hence, based on the above discussion, the hybrid interweave-underlay technique, the interweave technique, and the underlay technique, respectively, can fulfil these requirements for $6 \mathrm{G}$ by reusing the countrywide full $28 \mathrm{GHz}$ spectrum of all MNOs to in-building small cells of 
roughly $33.33 \%, 50 \%$, and $50 \%$ less number of buildings of small cells than that required by the SLSA technique.

In general, the required value of $L$ that can satisfy both $\mathrm{SE}$ and $\mathrm{EE}$ requirements for $6 \mathrm{G}$ varies with the value of $\omega$ since both $\mathrm{SE}$ and $\mathrm{EE}$ performances are a function of the product of them, i.e., $(\omega \times L)$. Specifically, SE and EE requirements for $6 \mathrm{G}$ can be obtained either by increasing $\omega$ with a corresponding decrease in $L$ or by decreasing $\omega$ with a corresponding increase in $L$. For example, for $\omega=12$, the required values of $L$ to satisfy SE and EE requirements for $6 \mathrm{G}$ are 1,2,2, and 3 , respectively, for the hybrid interweave-underlay, interweave, underlay, and SLSA techniques. In short, by trading off, the values of $L$ and $\omega$, the required SE and EE for $6 \mathrm{G}$ can be achieved.

\section{Conclusion}

In this paper, we have proposed power-domain based dynamic spectrum access (PDSA) techniques to share the $28 \mathrm{GHz}$ spectrum of one MNO with small cells in a building of another MNO in a country. The proposed PDSA techniques have explored the traditional interweave and underlay spectrum access techniques separately, as well as jointly, by controlling the transmission power of SBSs of each MNO in a country. Unlike [20], average capacity, spectral efficiency, energy efficiency, cost efficiency, and throughput per small cell UE performance metrics have been derived when the interweave and the underlay spectrum access techniques operate separately, as well as jointly, for an arbitrary number of MNOs countrywide. With numerical and simulation results, we have evaluated the performances of the interweave and underlay, as well as hybrid interweave-underlay spectrum access techniques, for an example scenario of four MNOs in a country.

It is shown that, due to gaining more shared spectra, the hybrid interweave-underlay technique improves all the above performance metrics considerably as compared to that of the interweave and underlay techniques when employing separately. Particularly, the maximum amount of the shared spectrum obtained by employing the hybrid interweave-underlay technique is 3 times (as opposed to 1.5 times the licensed spectrum for both interweave and underlay techniques) the licensed spectrum of any MNO for any observation time. This causes the hybrid interweave-underlay technique to improve the average capacity and SE, as well as throughput per small cell UE, by about 2.9 times in comparison with that of SLSA. These correspond to the improvement in these metrics by about 1.49 times and 1.4 times, respectively, when applying the interweave and underlay techniques separately. Further, the hybrid interweave-underlay technique improves the $\mathrm{EE}$ by about $73 \%$ and the CE by about $74.4 \%$ with respect to that of SLSA, which corresponds to the EE improvement by about $57.46 \%$ and $57.65 \%$, respectively, and the CE improvement by about $60 \%$ and $58.4 \%$, respectively, when applying the interweave and the underlay techniques separately.

Moreover, average capacity and hence SE increase linearly, whereas $\mathrm{EE}$ and $\mathrm{CE}$ improve negative-exponentially for all techniques as $L$ increases, and the hybrid interweaveunderlay technique provides the best, whereas the SLSA provides the worst, performances of all techniques in terms of average capacity, SE, EE, and CE. Furthermore, we have shown that the hybrid interweave-underlay technique, the interweave technique, and the underlay technique, respectively, can fulfill both spectral and energy efficiencies requirements for $6 \mathrm{G}$ networks by reusing the countrywide full $28 \mathrm{GHz}$ spectrum of all MNOs to in-building small cells of roughly $33.33 \%, 50 \%$, and $50 \%$ less number of buildings of small cells than that required by the SLSA for an intrabuilding spectrum reuse factor of six.

Overall, the required value of $L$ to obtain the expected spectral and energy efficiencies of $6 \mathrm{G}$ varies with the value of the intrabuilding reuse factor $\omega$ since both SE and EE performances are a function of the product of them, i.e., $(\omega \times L)$. Specifically, SE and EE requirements for $6 \mathrm{G}$ can be satisfied either by increasing $\omega$ with a corresponding decrease in $L$ or by decreasing $\omega$ with a corresponding increase in $L$ such that by trading off the values of $L$ and $\omega$, the required spectral and energy efficiencies of $6 \mathrm{G}$ can be achieved. The proposed technique can be investigated further for a complete analysis to address numerous crucial issues, including mmWave bands other than $28 \mathrm{GHz}$, such as $26 \mathrm{GHz}, 38 \mathrm{GHz}$, and $60 \mathrm{GHz}$; non-LOS path loss models; directional mmWave antennas; spectrum sensing mechanisms and control signaling overhead; implementation complexity analysis; burst traffic characteristics; and random deployments of indoor UEs; as well as serving more than one UE simultaneously by a single small cell in a building.

\section{Data Availability}

Data, primarily, in the form of numerous simulation assumptions and parameters reported previously by the standardization bodies, including 3rd Generation Partnership Project (3GPP) $[29,30]$ and International-Telecommunication Union-Radiocommunication Sector (ITU-R) [31], included and detailed within the article in Table 3 , were used to carry out the performance evaluation of this study. Other prior studies than these above [29-31] were cited at relevant places within the text as references [32-34, 36-39]. No data other than these were used to evaluate the performance studies. Taking into account all these parameters and assumptions, performance results were generated by a simulator running on a personal computer, which was built by the author using the standard computational tool MATLAB R2012b. MATLAB codes are not publicly available. However, supports for writing MATLAB instruction codes can be provided over the emails querying directly to the author at rony107976@gmail.com.

\section{Disclosure}

This paper was presented partly at the Fifteenth International Conference on Systems and Networks Communications (ICSNC), Porto, Portugal, 2020 [20].

\section{Conflicts of Interest}

The author declares that there is no conflict of interest regarding the publication of this paper. 


\section{References}

[1] Spectrum Policy Task Force Report, Federal Communications CommissionWashington, DC, USA2020, https://www.fcc.gov/ document/spectrum-policy-task-force.

[2] F. Mehmeti and T. Spyropoulos, "Performance analysis, comparison, and optimization of interweave and underlay Spectrum access in cognitive radio networks," IEEE Transactions on Vehicular Technology, vol. 67, no. 8, pp. 7143-7157, 2018.

[3] I. F. Akyildiz, Won-Yeol Lee, M. C. Vuran, and S. Mohanty, "A survey on spectrum management in cognitive radio networks," IEEE Communications Magazine, vol. 46, no. 4, pp. 40-48, 2008.

[4] A. Sharmila and P. Dananjayan, "Spectrum sharing techniques in cognitive radio networks - a survey," in The 2019 IEEE international conference on system, computation, automation and networking (ICSCAN), pp. 1-4, Pondicherry, India, 2019.

[5] M. G. Khoshkholgh, K. Navaie, and H. Yanikomeroglu, "Interference management in underlay spectrum sharing using indirect power control signalling," IEEE Transactions on Wireless Communications, vol. 12, no. 7, pp. 3264-3277, 2013.

[6] Z. Shi, X. Xie, and H. Lu, "Deep reinforcement learning based intelligent user selection in massive MIMO underlay cognitive radios," IEEE Access, vol. 7, pp. 110884-110894, 2019.

[7] W. Liang, K. D. Wang, J. Shi, L. Li, and G. K. Karagiannidis, "Distributed sequential coalition formation algorithm for spectrum allocation in underlay cognitive radio networks," IEEE Access, vol. 7, pp. 56803-56816, 2019.

[8] M. El Tanab and W. Hamouda, "Resource allocation for underlay cognitive radio networks: a survey," IEEE Communications Surveys \& Tutorials, vol. 19, no. 2, pp. 1249-1276, 2017.

[9] R. K. Saha, "Underlay cognitive radio millimeter-wave spectrum access for in-building dense small cells in multioperator environments toward 6G," in IEEE 23rd International Symposium on Wireless Personal Multimedia Communications (WPMC), Virtual Edition, pp. 105-110, Okayama, Japan, 2020.

[10] Y. Choi and D. Kim, "Power allocation and dimensioning for interweaved primary and secondary multicast networks with outage constraints," IEEE Communications Letters, vol. 18, no. 8, pp. 1415-1418, 2014.

[11] O. Simeone, Y. Bar-Ness, and U. Spagnolini, "Stable throughput of cognitive radios with and without relaying capability," IEEE Transactions on Communications, vol. 55, no. 12, pp. 2351-2360, 2007.

[12] A. Patel, M. Z. A. Khan, S. N. Merchant, U. B. Desai, and L. Hanzo, "The achievable rate of interweave cognitive radio in the face of sensing errors," IEEE Access, vol. 5, pp. 85798605, 2017.

[13] A. Kaushik, S. K. Sharma, S. Chatzinotas, B. Ottersten, and F. K. Jondral, "Sensing-throughput tradeoff for interweave cognitive radio system: a deployment-centric viewpoint," IEEE Transactions on Wireless Communications, vol. 15, no. 5, pp. 3690-3702, 2016.

[14] R. K. Saha, "Interweave shared-use model for dynamic spectrum access in millimeter-wave mobile systems for 6G," in 2020 IEEE 92nd Vehicular Technology Conference (VTC2020Fall), pp. 1-6, Victoria, BC, Canada, 2020.

[15] R. K. Saha, "A hybrid interweave-underlay countrywide millimeter-wave spectrum access and reuse technique for CR indoor small cells in 5G/6G era," Sensors, vol. 20, no. 14, p. $3979,2020$.
[16] A. U. Khan, G. Abbas, M. Tanveer, S. Ullah, and A. Naushad, "HBLP: a hybrid underlay-interweave mode CRN for the future 5G-based Internet of Things," IEEE Access, vol. 8, pp. 63403-63420, 2020.

[17] P. Zuo, T. Peng, W. Linghu, and W. Wang, "Optimal resource allocation for hybrid interweave-underlay cognitive Satcom uplink," in 2018 IEEE wireless communications and networking conference (WCNC), pp. 1-6, Barcelona, Spain, 2018.

[18] M. Jazaie and A. R. Sharafat, "Downlink capacity and optimal power allocation in hybrid underlay-interweave secondary networks," IEEE Transactions on Wireless Communications, vol. 14, no. 5, pp. 2562-2570, 2015.

[19] R. K. Saha, "Countrywide mobile spectrum sharing with small indoor cells for massive spectral and energy efficiencies in 5G and beyond mobile networks," Energies, vol. 12, no. 20, p. 3825, 2019.

[20] R. K. Saha, "Hybrid interweave-underlay millimeter-wave spectrum access in multi-operator cognitive radio networks toward 6G," in Fifteenth International Conference on Systems and Networks Communications (ICSNC), pp. 42-48, Porto, Portugal, IARIA, 2020.

[21] D. Chen, T. Jiang, and Z. Zhang, "Frequency partitioning methods to mitigate cross-tier interference in two-tier femtocell networks," IEEE Transactions on Vehicular Technology, vol. 64, no. 5, pp. 1793-1805, 2015.

[22] R. K. Saha, "A technique for massive spectrum sharing with ultra-dense in-building small cells in 5G era," in 2019 IEEE 90th Vehicular Technology Conference (VTC2019-Fall), pp. 1-7, Honolulu, HI, USA, 2019.

[23] A. Ali and W. Hamouda, "Advances on spectrum sensing for cognitive radio networks: theory and applications," IEEE Communications Surveys \& Tutorials, vol. 19, no. 2, pp. 1277-1304, 2017.

[24] J. Oh and W. Choi, "A hybrid cognitive radio system: a combination of underlay and overlay approaches," in 2010 IEEE 72nd Vehicular Technology Conference - Fall, pp. 1-5, Ottawa, ON, Canada, 2010.

[25] R. K. Saha, "A hybrid system and technique for sharing multiple spectrums of satellite plus mobile systems with indoor small cells in 5G and beyond era," IEEE Access, vol. 7, pp. 77569-77596, 2019.

[26] R. K. Saha and C. Aswakul, "A novel frequency reuse technique for in-building small cells in dense heterogeneous networks," IEEJ Transactions on Electrical and Electronic Engineering, vol. 13, no. 1, pp. 98-111, 2018.

[27] “Pascal's triangle. Wikipedia," October 2020, https://en .wikipedia.org/wiki/Pascal\%27s_triangle.

[28] R. K. Saha, "3D spatial reuse of multi-millimeter-wave spectra by ultra-dense in-building small cells for spectral and energy efficiencies of future $6 \mathrm{G}$ mobile networks," Energies, vol. 13, no. 7, article 1748, 2020.

[29] Evolved Universal Terrestrial Radio Access (E-UTRA), "Radio frequency (RF) system scenarios. document 3GPP TR 36.942, V.1.2.0, 3rd Generation Partnership Project, Jul. 2007," February 2020, https://portal.3gpp.org/desktopmodules/ Specifications/SpecificationDetails.aspx? specificationId $=2592$.

[30] "Simulation assumptions and parameters for FDD HeNB RF requirements. document TSG RAN WG4 (Radio) Meeting \#51, R4-092042, 3GPP, May 2009," February, 2020, https:// www.3gpp.org/ftp/tsg_ran/WG4_Radio/TSGR4_51/ Documents/. 
[31] "Guidelines for evaluation of radio interface technologies for IMT-2020. Report ITU-R M.2412-0 (10/2017), Geneva, 2017," February, 2020, https://www.itu.int/dms_pub/itu-r/ opb/rep/R-REP-M.2412-2017-PDF-E.pdf.

[32] R. K. Saha, P. Saengudomlert, and C. Aswakul, "Evolution toward $5 \mathrm{G}$ mobile networks-a survey on enabling technologies," Engineering Journal, vol. 20, no. 1, pp. 87-119, 2016.

[33] G. R. Maccartney, T. S. Rappaport, S. Sun, and S. Deng, "Indoor office wideband millimeter-wave propagation measurements and channel models at 28 and $73 \mathrm{GHz}$ for ultradense $5 \mathrm{G}$ wireless networks," IEEE Access, vol. 3, pp. 23882424, 2015.

[34] R. K. Saha, S. Nanba, and K. Nishimura, "A technique for cloud based clustering and spatial resource reuse and scheduling of 3D in-building small cells using CoMP for high capacity CRAN,” IEEE Access, vol. 6, pp. 71602-71621, 2018.

[35] R. K. Saha, "Spectrum allocation and reuse in $5 \mathrm{G}$ new radio on licensed and unlicensed millimeter-wave bands in indoor environments," Mobile Information Systems, vol. 2021, Article ID 5538820, 21 pages, 2021.

[36] Z. Zhang, Z. Ma, M. Xiao et al., "6G wireless networks: vision, requirements, architecture, and key technologies," IEEE Vehicular Technology Magazine, vol. 14, no. 3, pp. 28-41, 2019.

[37] S. Chen, S. Sun, S. Kang, W. Cheng, M. Peng, and M. Peng, "Vision, requirements, and technology trend of 6G: how to tackle the challenges of system coverage, capacity, user datarate and movement speed," IEEE Wireless Communications, vol. 27, no. 2, pp. 218-228, 2020.

[38] C.-X. Wang, X. Gao, X. H. You et al., "Cellular architecture and key technologies for $5 \mathrm{G}$ wireless communication networks," IEEE Communications Magazine, vol. 52, no. 2, pp. 122-130, 2014.

[39] G. Auer, C. Desset, I. Godor et al., "How much energy is needed to run a wireless network?," IEEE Wireless Communications, vol. 18, no. 5, pp. 40-49, 2011. 